\title{
Light and Organisms
}

\author{
Orienting Effects of Monochromatic Light on Fucus Spores and Rhyzoids \\ By Annie May Hurd, University, California
}

ONE of the most striking of the biological phenomena resulting from the action of light on organisms is the orientation of the first cleavage plane of germinatin spores by unilateral illumination. Whenever such illumination is sufficiently intense the first cross wall forms perpendicular to the direction of the incident light. This phenomenon has been demonstrated in Equisetum, Puccinia, Fucus and other algae 4, 8, , 10, together with the fact that the cell on the shaded side of the spore becomes the rhizoidal cell irrespective of gravity. Thus in these and related forms the polarity of the plant is established by the direction of light stimuli.

The power of light waves to so orient the plant is without doubt, the power to orient the spindle of the first dividing nucleus. The mechanics of such reactions may long remain unknown; but we have a sug gestive and possibly the ultimate explanation in Child's ${ }^{2}$ metabolic gradient theory. He has demonstrated in many marine plants and in some of the lower animals the existence of the so-called "axial
gradients." By an axial gradient is meant the decraesing rate of metabolic processes from the apical to the basal end. We may suppose that such a gradient is produced within a germinating spore wheneve there is sufficient difference in the amount of ligh energy received on two opposite sides to produce the requisite difference in the rate of the oxidation processes along the line of direction of illumination. If Child's supposition is correct, the cell on the shaded side of the spore becomes the rhizoidal cell by virtue of the fact that the least rapid rate of the oxidation reactions along the gradient determines the basal end, the most rapid the apical end, of the organism.

The purpose of the present investigation was to study the power of pure monochromatic lights to establish the polarity of the germinating spores of Fucus in flatus, and also to answer several questions concerning the negative phototropism of the young rhizoids; viz the determination of the exact wave lengths responsi ble for the phenomenon; the relative importance of the quality and quantity factors in the illumination or the role of intensity of illumination apart from the kind of light; and whether all effective monochromatic lights produce the same result as white light.

To obtain the monochromatic light, seven Wratten filter screens were used, each transmitting a narrow range of wave lengths but altogether em!racing the whole of the visible spectrum. The wave lengths to which each screen was transparent were determined by testing the light transmitted by each with a direct vision spectroscope with a wave length scale attached. These screens were then fitted as windows in the ends of boxes painted black on the inside. Culture dishe were made by cementing together microscope slides, so that the light entering the boxes through the screens fell on a flat side of the dish and thus entered the water normally with a minimum loss from reflection and refraction.

The electric arc was used as the source of light wherever possible because it gives all the desired wave lengths, with the result that the whole set of screen could be used in the same exposure, insuring for al the boxes identical conditions of temperature and duration of illumination.

The spectroscopic analysis of the light transmitted by the screens gave the exact wave lengths which would act on the cultures behind them. The next step was to devise a means of making the intensities of the light acting in each box equal so as to eliminate that mos mportant, and hitherto largely overlooked, factor in light reactions. This done, variations in results $n \mathrm{~b}-$ tained behind different screens might safely be attrib uted to differences in the quality of the stimulus. There have been several methods devised by which the relative intensities of monochromatic lights can be measured and made equal.1. $3.6,6,7$ But all of these in volve special apparatus not available for use in this

* From Proc. Nat'l. Acad. Sct. (Cambridge. Mass.)

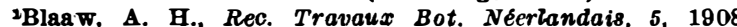
209-372)

Child, C. M., Individuallty of Organigms, 1915. Day, E. C., Bull. Mus. Comp. Zool. 5s, 1911 (303-343).
Frarmer, J. B., and Williams, J. L., Proc. Roy. Boc., $1896(188-195)$

Knlep, G. and Minder, F., Zs. Bot., 1, 1909 (619).

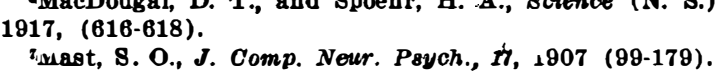

Nast, S. O., J. Comp. Neur. Psych., H, 1907 (99-179), (321-350). investigation; so a simpler method was devised where-
by the relative intensities of the lights transmitted by the Wratten filter-screens were measured by means of a thermopile and galvanometer and made equal by varying the distances of the dark boxes from the electric arc such that at these distances the defiections of the galvanometer, when the thermopile was exposed in turn to the light behind each filter screen, were equal. This distance was also measured with a piece of white glass as a filter screen which represented the removal of the control culture from the arc. The instruments used in this energy calibration were a Hilger thermopile and a d'Arsonval galvanometer.

It seems necessary on account of the questions which have been raised during the course of this work, to state here that the thermopile is equally sensitive to the energy of the red and of the violet ends of the spectrum, and is, therefore, an accurate measure of the total amount of light acting behind each color screen. The difference between heat and light is only a matter of wave length. The thermopile measures light in terms of the electric current produced by the difference in temperature of the exposed and unexposed junctions; but it does so by virtue of the fact that the energy of whatever vibrations fall upon it, be they long and therefore heating in their physiological effect, or short and therefore perceived as light, is converted into heat energy upon being absorbed by the exposed junction of the thermopile. In other words, the light of the blue end of the spectrum produces an electromotive force much less than that of the infra-red but no less measurable.

Once these distances from the arc, at which the intensities of the light in each box are equal, are deter mined, the quantity of light energy can be varied by multiplying or dividing all the distances by the same multiple and the intensities in all will still remain equal to each other. By means of a photometer the actual amount of light acting in each box can then be determined by measuring the intensity in candle meters behind the white glass control at the proper distance from the arc. Then from the law of inverse squares, viz., that the intensity of light per unit sur face varies inversely as the square of the distance from the source, the absolute intensity at any distance from the arc can be computed. So a Sharpe-Millar photometer was used to measure the intensity of the naked arc at the distance of the white light control. But it was then necessary to correct the measurements so obtained for the absorption of light by the glass of the filters. This so-called absorption coefficient was obtained by measuring with a Lummer Brodhum photometer the intensity of a light both with and without a screen of clear glass equal in thickness to that of the filters. It was found that glass $1.5 \mathrm{~mm}$. thick absorbed $12 \%$ of the light falling upon it. Therefore
to obtain the intensity of the light actually entering each dark box, it was necessary to take $88 \%$ of the reading given by the photometric measurement of the unscreened arc at the previously determined distance of the white light control.

The following table lists the colored screens used with the wave lengths they transmitted and the distances from the arc at which they were placed to make the intensity of light behind each equal to 1,800 meter candles. The lack of agreement between these values and the energy curve of the spectrum is due to the individual absorption of the filters and also to the fact that they do not all transmit the same number of wave lengths.

Table showing distances at which the intensities of light from an electric arc transmitted by Wratten light filters are equal

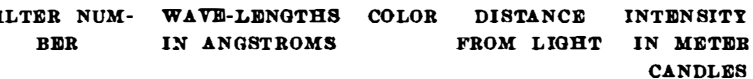

\begin{tabular}{|c|c|c|c|c|}
\hline & & & & \\
\hline 70 & $6600-7000$ & Red & 320 & 1800 \\
\hline & $6200-6800$ & Red & 275 & \\
\hline 72 & $5900-6200$ & Orange & 230 & 1800 \\
\hline 73 & $5600-5900$ & Yellow & 250 & 1800 \\
\hline 74 & $5200-5.600$ & Green & 280 & 1800 \\
\hline 75 & $4700-5200$ & Blue & 250 & 1800 \\
\hline & $4000-4700$ & Violet & 250 & 1800 \\
\hline atrol & $4000-7000$ & White & 340 & 1800 \\
\hline
\end{tabular}

To obtain the spores of Fucus inflatus for the experiments, the fruiting plants were collected at low tide, 'Rosenvinge, M. L. K., Revue Gen. Bot., 1, 1889 (125-135) vWinkler, E., Totd, 18, 1800 (297-308). kept over night in damp newspapers, and the next morning were dried slightly by exposing them to the air for about half an hour. Then when the fruiting tips were submerged in sea water in the culture dishes, large numbers of eggs and sperms were extruded and settled to the bottom of the dish. After removing the piece of plant the culture dish was placed in one of the little racks made to fit in the dark boxes behin the filter screens. The illumination of the culture was continued six to eight hours, this time having been found more than enough to cause the first cleavage plane to be permanently oriented regardless of subsequent illumination.

In the experiments for which the naked arc was the source of light, the heating effect was so great that the spores were killed very quickly. The mercury vapor lamp was next used to obtain wave lengths of the blue end of the spectrum and a 100-watt nitrogen flled Tungsten globe for the red. But as in the case of the electric arc, the Tungsten light killed the spores by the high temperature produced at the distances where it was necessary to place the cultures for a suffciently intense illumination. With the mercury vapor lamp, however, positive results were secured. The wave lengths which were found to produce the orientation of the first cleavage plane such that all the firs cross walls formed perpendicular to the direction of the incident rays, are those between 4000 and 5200 angstrom units. Behind the two other filter screens used with the mercury vapor lamp and transmitting wave lengths of 5200 to 5900 Angstrom units, the spores germinated as if in darknéss with the orientations of the first cleavage planes following no rule, and the rhizoids extending in all directions. Howeve the intensities of the lights behind these color screen were not equal when the mercury lamp was used be cause the shorter blue wave lengths predominated to so great a degree and hence produced greater intensities With regard to the phototropism of the young rhizoids, it was found that very weak white light, too weak to orient the cleavage planes, would cause the growing tips to turn sharply away from the source of light. With the intensity of illumination behind all the color screens equal to 1800 meter candies, only the blue and violet lights produced the phototropism. The other wave lengths at this intensity had no effect, the young rhizoids continuing in the direction in which they had started just as did those of the control in darkness. However when a more intense illumination was secured by placing the boxes in direct sunlight, the rhizoids behind the green filter, in addition to those behind the blue and violet ones, showed the same negative phototropism. This and subsequent experiments would lead us to believe that both quantity and quality, or intensity and wave length, are determining factors in the power of light stimuli to produce phototropisms.

In every culture of Fucus inflatus whether germi nated in darkness or in strong unilateral light a most striking orientation of the first cross-wall with reference to adjacent spores appears. Wherever a group of spores are lying within about $0.2 \mathrm{~mm}$. of each other, the first cleavage plane is perpendicular to the direction of the center of the group. The cell toward the interior invariably becomes the rhizoidal cell. This phenomenon was reported by Rosenvinge $e^{\bullet}$ in other species of Fucus and in Ascophyllum. For want of a better term I have called it group orientation. A study of the phenomenon was made to determine the strength of this stimulus, compared to that of light, in its power to establish the orientation of the plant. It was at once very evident that for the most spores the former stimulation is stronger when the spores are within a short distance of each other-- $0.2 \mathrm{~mm}$. or often morebut beyond this distance, the chemical stimulus becomes too weak and only the light is able to determine the polarity of the plant. Only the comparatively isolated spores therefore show the orientation to light with the sources of illumination used here. The phenomenon is very conspicuous in groups of 2,3 or 4 eggs as well as in masses of 50 or 100 . In these large groups it is made evident by the invariable rule that no rhizoid ever extends outward from a group. When two spores are within the distance through which the stimulus is effective, the first cleavage planes of the two are parallel and the rhizoids grow towards each other and often meet tip to tip. The groups of 5 or 6 often make symmetrical star-like designs when the (Continued on page 178) 